EESTI NSV TEADUSTE AKADEEMIA TOIMETISED. 29. KOIDE FUUSIKA * MATEMAATIKA. 1980, NR. 1

ИЗВЕСТИЯ АКАДЕМИИ НАУК ЭСТОНСКОИ ССР. ТОМ 29 ФИЗИКА * МАТЕМАТИКА, 1980, Ni 1

\title{
ТОНКОСТРУКТУРНЫЕ ВИБРОННЫЕ СПЕКТРЫ МОЛЕКУЛ ХЛОРОФИЛЛА И ЕГО ПРОИЗВОДНЫХ В ТВЕРДЫХ РАСТВОРАХ ПРИ ВОЗБУЖДЕНИИ ПЕРЕСТРАИВАЕМЫМ ЛАЗЕРОМ НА КРАСИТЕЛЕ
}

\author{
(Представлена ,К. К. Ребане)
}

\begin{abstract}
Измерены спектры флуоресценции и возбуждения флуоресценции замороженных растворов хлорофилла $a$, протохлорофилла, феофитина $a$ и его $\mathrm{Zn}$-комплекса при $T=4,2 \mathrm{~K}$ c использованием перестраиваемого лазерного возбуждения. На основе тонкой внбронной структуры определен ряд колебательных частот этой группы родственных молекул.
\end{abstract}

\section{Введение}

Флуоресценция хлорофилла, как функция его состояния in vivo, широко применяется для получения информации о первичных фотохимических процессах в фотосинтезе (см., напр., [ $\left.{ }^{1}\right]$ ). Спектральные исследования флуоресценции и поглощения важны для изучения электронно-колебательных взаимодействий в возбужденных электронных состояниях хлорофилла и его производных, а также для выяснения механизмов взаимодействия с молекулами растворителя.

В то время как при комнатной и азотной температурах спектры флуоресценции и поглощения хлорофилловых растворов состоят из сравнительно широких полос $\left(\approx 300 \mathrm{~cm}^{-1}\right)$, при температуре жидкого гелия $\left(4,2\right.$ K) возможно проявление квазилинейчатой структуры $\left[{ }^{2,3}\right]$. Оказалось, что применение монохроматического возбуждения позволяет наблюдать квазилинейчатую структуру в спектре флуоресценции хлорофилла $a$ (Хл $a)$ практически в любом растворителе $\left[{ }^{4}\right]$. Впоследствии тонкая структура была обнаружена и для некоторых производных Хл $a\left[{ }^{5,6}\right]$.

Возникновение квазилинейчатой структуры в спектре флуоресценции Хл $a$ объясняется $\left[{ }^{4,5}\right]$ аналогично обнаруженному в [ $\left.{ }^{7}\right]$ снятию неоднородного уширения с помощью монохроматического возбуждения - преимущественно возбуждаются лишь те молекулы, бесфононная линия которых попадает в резонанс с возбуждающей лазерной линией. * Однако особенностью хлорофиллоподобных молекул является то, что возбуждение охватывает сразу ряд близкорасположенных (в пределах неоднородной ширины) электронно-колебательных (виброн-

\footnotetext{
* В случае т. н. систем Шпольского само неоднородное распределение содержит узкие пики, обеспечивающие квазилинейчатую структуру даже при «белом» возбуждении. Для хлорофилла такие условия, по-видимому, не реализуются $\left[{ }^{6}\right]$.
} 
ных) переходов в спектре поглощения. Это приводит к умножению числа бесфононных линий спектра флуоресценции (подобная особенность спектров флуоресценции экспериментально наблюдалась для ряда молекул $\left[{ }^{8,9}\right]$, элементарная теоретическая трактовка дана в $\left.\left[{ }^{10}\right]\right)$.

Основным достоинством таких многолинейчатых спектров является возможность определения из спектра флуоресценции частот внутримолекулярных колебаний в возбужденном электронном состоянии. Использование лазера на красителе с перестраиваемой частотой позволяет охватывать достаточно широкий интервал частот (в случае Хл $a$ и Хл $b$ удалось перекрыть всю область внутримолекулярных колебаний $\left.\left[{ }^{11}\right]\right)$. Все же более непосредственно частоты можно определить из спектра возбуждения при монохроматической регистрации. Благодаря применению сканируемого лазерного излучения вместо возбуждения через монохроматор, нам удалось довести спектральное разрешение в настоящей работе до $1 \mathrm{~cm}^{-1}$ против $7 \mathrm{~cm}^{-1}$ в [ [5]. На основе полученных частот провели сравнительное изучение наиболее близких родственных соединений Хл $a$ : протохлорофилла (ПХл), феофитина $a$ (Фео $a$ ) и его Zn-комплекса $(\mathrm{Zn}-\Phi$ ео $a)$, а также Хл $b$.

\section{Методика эксперимента}

Хл $a$ и Хл $b$ выделили из листьев крапивы по известному методу хроматографии на колонке [12], их феофитины - путем обработки $\mathrm{HCl}$ и дальнейшего хроматографирования. Zn-Фео $а$ получили из Фео $а$ в уксусной кислоте с добавлением Zn-ацетата путем кипячения при $70{ }^{\circ} \mathrm{C}$. Продукт реакции экстрагировали эфиром, добавляя воду, после чего провели хроматографирование на сахарной колонке. ПХл выделили из семян тыквы. Растворы пигментов в эфире (Э) и в смеси эфир-бутанол $1: 1$ (ЭБ) использовались при концентрациях от $10^{-6}$ до $10^{-5}$ моль/л. Качество пигментов контролировалось по спектрам поглощения на спектрофотометре «Acta MVII».

Спектральные измерения проводились при возбуждении лазером на красителе CR 490 (родамин 6Ж перекрывал область от 585 до 630 нм), который накачивался аргоновым лазером «Spectra-Physics», модель 171 . Ширина линии генерации была меньше $1 \mathrm{~cm}^{-1}$. Флуоресценция регистрировалась на спектрометре ДФС-24 со спектральным разрешением $0,5 \mathrm{CM}^{-1}$.

\section{Результаты и обсуждение}

Характерным примером квазилинейчатой структуры при возбуждении в области вибронного поглощения $S_{1} \leftarrow S_{0}$ может служить спектр флуоресценции $\mathrm{Zn}$-Фео $a$ на рис. 1. Так как преобладает неоднородное уширение, то при монохроматическом возбуждении наблюдается эффект размножения линий $\left[{ }^{8}\right]$. Все линии в спектре флуоресценции в сущности чисто-электронны, поскольку взаимодействие с колебаниями основного состояния весьма слабо. Поэтому интервалы от возбуждающей линии до линий свечения соответствуют частотам колебаний $\omega^{\prime}$ в электронном состоянии $S_{1}$. Ширина наиболее узких линий составляет $5 \mathrm{~cm}^{-1}$, что значительно превышает ширину аппаратной функции, т. е. имеется остаточное неоднородное уширение колебательных уровней. Из-за того, что возбуждение резонансно не с $0-0$-переходом, а с определенными вибронными переходами, однородная ширина, как правило, при такой методике не достигается $\left[{ }^{10,}{ }^{3}\right]$. 

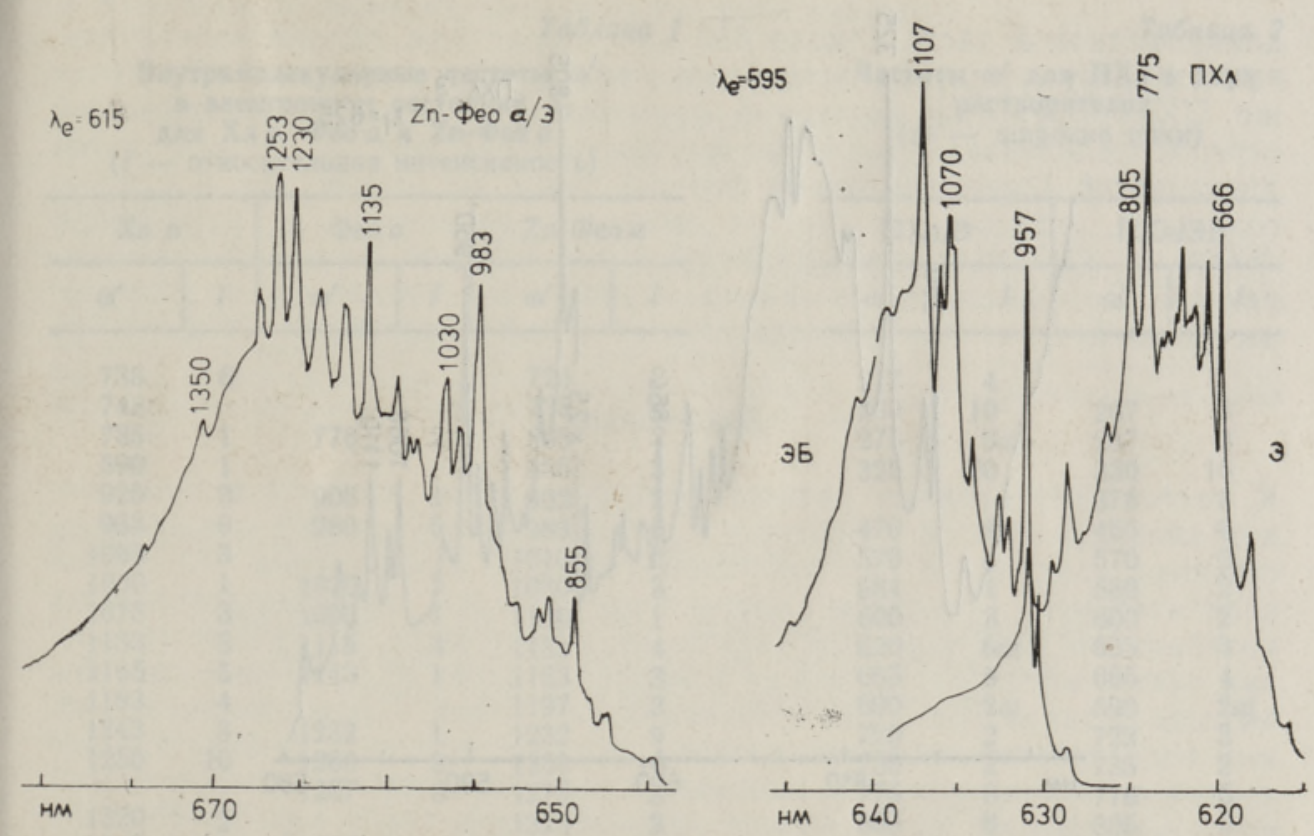

Рис. 1. Спектр флуоресценции $\mathrm{Zn}$-Фео $a$ в эфире при монохроматическом лазерном возбуждении на частоте $v_{e}=$ $=16256 \mathrm{~cm}^{-1}$. Около линий указаны вычисленные из спектра частоты колебаний. $T=4,2 \mathrm{~K}$ на всех рисунках.

Рис. 2. Спектры флуоресценции ПХл в эфире (справа) и в смеси эфир - бутанол (слева) при возбуждении $v_{e}=$ $=16802 \mathrm{~cm}^{-1}$.

Рис. 3. Спектры возбуждения флуоресценции Фео $a$ (внизу, час-

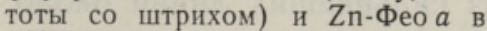
эфире при регистрации $v_{f}=$ $=15034$ и $15263 \mathrm{~cm}^{-1}$ соответст. венно.

Впервые удалось получить также квазилинейчатый спектр флуоресценции ПХл (рис. 2). Тонкость заключалась в том, что при возбуждении в состояние $S_{2}$ или выше тонкая структура отсутствует [ $\left.{ }^{6}\right]$, очевидно, из-за недостаточной корреляции между энергиями разных состояний $\left[{ }^{14}\right]$.

Богатая структура наблюдалась в спектрах возбуждения (рис. 3 и 4), из которых были определены колебательные частоты $\omega^{\prime}$ в наиболее актуальной высокочастотной обла-

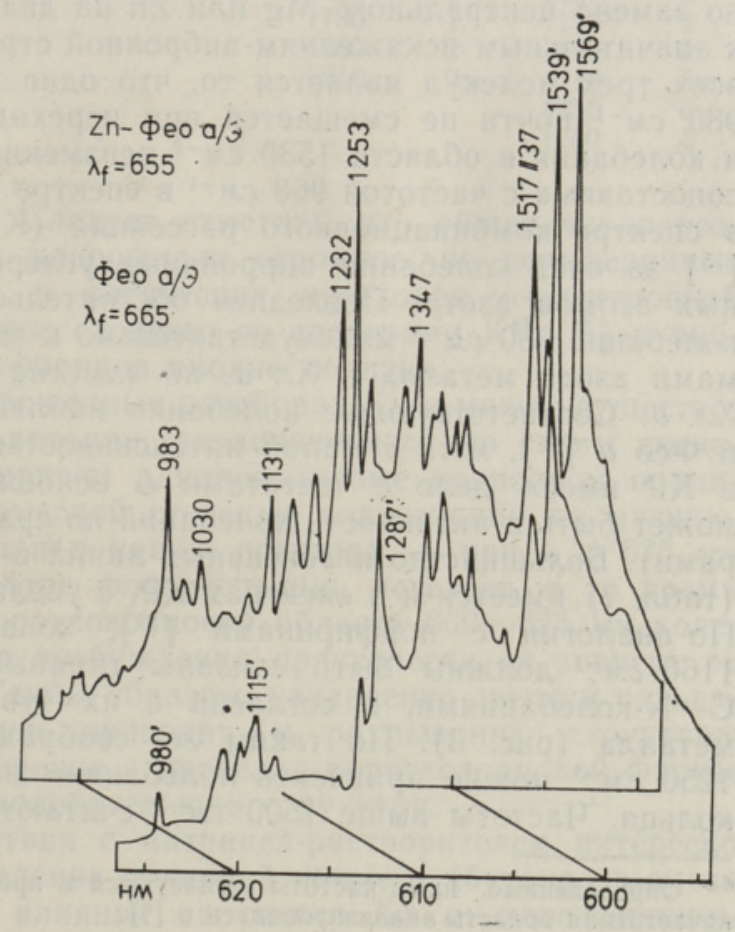




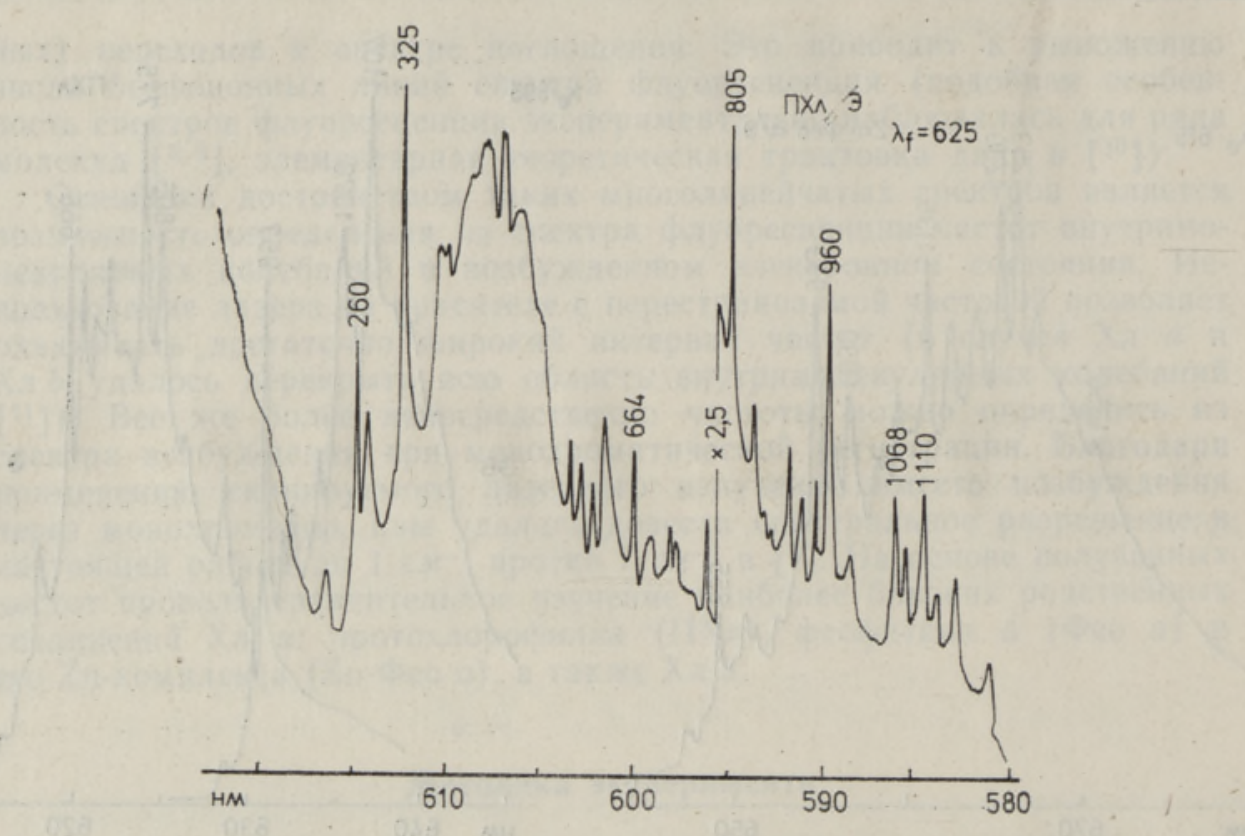

Рис. 4. Спектр возбуждения ПХл в эфире при регистрации $v_{f}=15996 \mathrm{cs}^{-1}$.

сти**. Частоты, средние из 2-4 спектров при разных длинах вфлн регистрации, сведены в табл. 1 и 2. Точность определения частот $2 \mathrm{CM}^{-1}$.

Частоты Хл $a$ и $\mathrm{Zn}$-Фео а достаточно хорошо сопоставимы (табл. 1), но замена центрального $\mathrm{Mg}$ или $\mathrm{Zn}$ на два водорода в Фео $a$ приводит к значйтельным искажениям вибронной структуры (рис. 3). Общим для всех трех молекул является то, что одна из сильнейших линий около $980 \mathrm{~cm}^{-1}$ почти не смещается при переходе от молекулы к молекуле и колебания в области $1530 \mathrm{~cm}^{-1}$ неизменно активны. Частота $980 \mathrm{~cm}^{-1}$ сопоставима с частотой $960 \mathrm{~cm}^{-1}$ в спектре ПХл и с аналогичным пиком в спектре комбинационного рассеяния (KР) порфиринов, отнесенным $\left[{ }^{15}\right]$ за счет колебания пиррольных углеродов, без участия центральных атомов азота. Последнее обстоятельство согласуется с тем, что колебание $980 \mathrm{~cm}^{-1}$ малочувствительно к замещениям связанного с атомами азота металла в Хл $a$, но заметно изменяегся при переходе к Хл $b$. Соответствующее колебание наблюдалось в спектре КР Хл $a$ и Фео $a\left[{ }^{16}\right]$, хотя с малой интенсивностью (не следует забывать, что в $\mathrm{KP}$ имеют дело с частотами $\omega$ основного состояния $S_{0}$, различна может быть и активность колебаний по сравнению с вибронными спектрами). Большинство интенсивных линий в области начиная с $1130 \mathrm{~cm}^{-1}$ (табл. 1) имеется и в спектрах $\mathrm{KP}$, с увеличением частот на $\approx 10 \mathrm{~cm}^{-1}$. По аналогии с порфиринами $\left[{ }^{15}\right]$, колебания в интервале 1130 $1160 \mathrm{~cm}^{-1}$ должны быть связаны главным образом с пиррольными $\mathrm{C}-\mathrm{N}$-колебаниями, в согласии с их чувствительностью к удалению металла (рис. 3). По таким же соображениям группу линий около $1250 \mathrm{~cm}^{-1}$ можно приписать колебаниям внутренней зоны порфинового кольца. Частоты выше $1500 \mathrm{~cm}^{-1}$ считаются включающими колебания

** Определенные нами частоты согласуются в пределах $5 \mathrm{~cm}^{-1}$ с данными [ ${ }^{11}$ ]. Низкочастотная область анализировалась в [ $\left.{ }^{6}\right]$. 
Таблица 1

Внутримолекулярные частоты $\omega^{\prime}$ в электронном состоянии $S_{1}$ для Хл $a$, Фео $a$ и $\mathrm{Zn}-\Phi$ ео $a$ ( $I$ - относительная интенсивность)

\begin{tabular}{|c|c|c|c|c|c|}
\hline \multicolumn{2}{|c|}{ Хл $a$} & \multicolumn{2}{|c|}{ Феo $a$} & \multicolumn{2}{|c|}{$\mathrm{Zn}-\Phi$ eo $a$} \\
\hline$\omega^{\prime}$ & $I$ & $\omega^{\prime}$ & $I$ & $\omega^{\prime}$ & $I$ \\
\hline
\end{tabular}

$\begin{array}{rrrrrr}738 & 6 & & & 725 & 2 \\ 748 & & & & 735 & 6 \\ 785 & 1 & 770 & 2 & 805 & 2 \\ 890 & 1 & & & 855 & 3 \\ 925 & 3 & 906 & 1 & 902 & 2 \\ 983 & 9 & 980 & 5 & 983 & 6 \\ 1005 & 3 & & & 1010 & 2 \\ 1030 & 1 & 1070 & 2 & 1030 & 3 \\ 1075 & 3 & 1090 & 1 & 1100 & 1 \\ 1133 & 5 & 1115 & 3 & 1133 & 4 \\ 1165 & 5 & 1143 & 1 & 1163 & 3 \\ 1193 & 4 & & & 1197 & 3 \\ 1243 & 3 & 1232 & 1 & 1232 & 9 \\ 1250 & 10 & 1260 & 3 & 1253 & 10 \\ & & 1287 & 5 & 1275 & 3 \\ 1320 & 2 & & & 1325 & 3 \\ 1345 & 10 & 1357 & 4 & 1347 & 4 \\ 1372 & 7 & 1378 & 4 & 1370 & 2 \\ 1397 & 3 & 1400 & 1 & 1404 & 3 \\ 1415 & 2 & 1420 & 2 & 1422 & 3 \\ 1510 & 10 & 1508 & 3 & 1515 & 10 \\ 1530 & 5 & 1539 & 10 & 1537 & 6 \\ & & 1569 & 10 & 1655 & 1 \\ 1655 & 1 & & & 1655 & 1\end{array}$

Таблича 2

Частоты $\omega^{\prime}$ для ПХл в двух растворителях

(ш - широкие пики)

\begin{tabular}{|c|c|c|c|}
\hline \multicolumn{2}{|c|}{ пХл/Э } & \multicolumn{2}{|c|}{ ПХл/ЭБ } \\
\hline$\omega^{\prime}$ & $I$ & $\omega^{\prime}$ & $=3-I$ \\
\hline
\end{tabular}

$\begin{array}{rccc}217 & 4 & & \\ 260 & 10 & 267 & 3 \\ 275 & 6 \amalg & 287 & 3 \\ 325 & 20 & 330 & 10 \\ & & 375 & 3 \\ 470 & 6 & 466 & 4 \\ 570 & 4 & 570 & 2 \\ 584 & 4 & 580 & 2 \\ 600 & 3 & 600 & 2 \\ 620 & 6 \mathrm{~m} & 625 & 3 \\ 665 & 8 & 665 & 4 \\ 690 & 2 \mathrm{~W} & 690 & 2 \mathrm{~m} \\ 720 & 2 & 723 & 3 \\ 730 & 2 & 735 & 2 \\ 775 & 8 & 776 & 5 \\ 805 & 8 & 805 & 5 \\ 845 & 2 & 845 & 1 \\ 905 & 3 & 895 & 2 \\ 920 & 3 & 934 & 3 \\ 943 & 3 & & 4 \\ 960 & 9 & 957 & 10 \\ & & 982 & 1 \\ 1000 & 1 & 993 & 1 \\ 1068 & 3 & 1035 & 1 \\ 1085 & 2 & 1070 & 4 \\ 1110 & 3 & 1107 & 5 \\ 1130 & 1 & 1135 & 2 \\ 1160 & 2 & 1150 & 3 \\ 1210 & 2 & 1210 & 8 \amalg \\ & & 1235 & 6 \\ & & 1260 & 3 \\ & & & \end{array}$

метиновых мостиков $\left[{ }^{15,16}\right]$. Следует отметить, что общее число возможных колебаний молекул хлорофилла огромное, но периферийные группы заведомо неактивны в оптических переходах $\pi$-электронной системы. Поэтому качественное сходство со спектрами КР $\left[{ }^{15}\right]$ и вибронными спектрами [ $\left.{ }^{17}\right]$ порфиринов вполне понятно.

Известно $\left[{ }^{18}\right]$, что в замороженных растворах Хл $а$ может существовать в разных формах сольватации, различающихся по своим спектральным характеристикам, причем длинноволновые подполосы приписываются формам с более высокой степенью сольватации по магнию. Две формы обнаруживаются и в наших спектрах - при $\lambda>675$ н.м линейчатая структура в спектре флуоресценции исчезает, в то время как для флуоресценции в коротковолновой области $660-675$ нм колебательная структура спектра возбуждения практически не зависит от длины волны регистрации. Таким образом, увеличение степени сольватации Хл $a$ с растворителем приводит к размыванию структуры спектра, а неоднородное уширение в пределах коротковолновой формы сольватов мало влияет на колебательную структуру.

В отношении взаимодействия с матрицей-растворителем интересно сравнить Хл $a$ и ПХл. Добавление полярной примеси (бутанол в смеси ӘБ) не оказывало особого влияния на спектр Хл $a$ : интенсивность 
длинноволновой части (около 680 нм) возрастала, однако колебательная структура спектров оставалась практически неизменной. В случае же ПХл добавление полярной примеси приводило к значительному перераспределению в спектре флуоресценции (рис. 2). Здесь линии дисольватов (длинноволновой формы) такие же четкие, как и у моносольватов, однако ряд частот оказался различным у двух форм (табл. 2). Следовательно, механизмы сольватации Хл $a$ и ПХл неодинаковы. Возможно, эти различия объясняются образованием водородных связей через два дополнительных атома водорода у Хл $a$.

\section{Заключение}

К настоящему времени решены принципиальные вопросы возникновения квазилинейчатой структуры в вибронных спектрах Хл $a$ и его родственных соединений при монохроматическом возбуждении. На основе колебательной структуры определены частоты внутримолекулярных колебаний в возбужденном электронном состоянии. На основе сравнения частот молекулы Хл $a$ и ее наиболее близких производных сделаны выводы о характере соответствующих колебаний. Продолжение подобных исследований позволит, с одной стороны, получить данные о более тонких аспектах внутри- и межмолекулярного взаимодействия, а с другой, - разрешить такие весьма важные для данного класса объектов вопросы, как чисто однородная ширина линий и скорость колебательной релаксации.

Авторы искренне благодарны К. К. Ребане за обсуждение результатов настоящей работы.

\section{ЛИТЕРАТ У РА}

1. Lavore 1, J., Etienne, A.-L., In: Topics of Photosynthesis, 2, Primary Processes of Photosynthesis, Amsterdam, Elsevier, 1977, p. 203-268.

2. Ли т вин Ф. Ф., Пер сонов Р. И., Коротаев О. Н., Докл. АН СССР, 188, № $5,1169-1171$ (1969).

3. Ав а рма а Р., Реба н е К., Изв. АН ЭССР, Физ. Матем., 22, № 1, 108-110 (1973)

4. А в а рм а а Р., Изв. АН ЭССР, Физ. Матем., 23. № 1. 93-94 (1974).

5. A v a rm a a, R., R e b a ne, K., Stud. biophys., 48, № 3, 209-218 (1975).

6. А в а рм а а Р. А., М а ур ин г К. Х., Ж. прикладной спектроскопии, 28 , № 4, $658-662(1978)$

7. Персонов Р. И., А льши ц Е. И., Быковская Л. А., Письма в ЖЭТФ, 15, вып. 10, 609-612 (1972)

8. Ребане К. К., А в а рм а а Р. А., Гороховский А. А., Изв. АН СССР, Сер. физ., 39, № 9, 1793-1800 (1975).

9. Альшиц Е. И., Персонов Р. И., Стогов В. И., Изв. АН СССР, Сер. физ., 39, № 9, 1918-1921 (1975)

10. А в а р м а а Р., Изв. АН ЭССР, Физ. Матем., 23, № 3. $238-247$ (1974).

11. Fünfschilling, J., Willi ams, D. F., Photochem. Photobiol., 26, № 1, 109113 (1977).

12. Тын и ссоо В., Т а мк и ви Р., Изв. АН ЭССР, Хим., 27. № 4, 219-223 (1978).

13. Gorokhovski, A. A., Kikas, J., Opt. Commun., 21, № 2, $272-274$ (1977).

14. С а а р и П. М., Т а м м Т. Б., Изв. АН СССР, Сер. физ., 39, № 11, 2321-2325 (1975).

15. Гладков Л. Л., Градюшко А. Т., Ксенофонтова Н. М., Соловь. е в К. Н., С т а р у хин А. С., Ш ульга А. М., Ж. прикладной спектроскопии, 27, № 3, 506-513 (1977).

16. L ut z, M., J. Raman Spectrosc., 2, № 5, 497-516 (1974)

17. Arabei, S. M., Shkirman, S. F., Solovyov, K. N., Yegorova, G. D., Spectrosc. Lett., 10, № 9, 677-697 (1977).

18. Hevesi, J., S ing ha l, G. S., Spectrochim. acta, 25A, № 11, 1751-1758 (1969).

Ннститут физики

Академии наук Әстонской ССР
Поступила в редакцию 10/IX 1979 
R. AVARMAA, R. TAMKIVI, Sirje KIISLER, Vaimi NOMM

\title{
KLOROFULLI JA LÄHEDASTE OHENDITE PEENSTRUKTUURSED VIBROONSPEKTRID TAHKETES LAHUSTES OMBERHÄALLESTATAVA VARVLASERIGA ERGASTAMISEL
}

On mõōdetud $a$-klorofülli, protoklorofülli, $a$-feofütiini ja selle $Z n$-kompleksi fluorestsentsi- ja ergastusspektreid külmutatud lahustes temperatuuril $4,2 \mathrm{~K}$, kasutades ümberhäälestatavat laserergastust. Vibroonsest peenstruktuurist on määratud rida nimetatud ühenditerühma vōnkesagedusi. On vōrreldud eri molekulide sagedusi omavahel ja porfüriinide sagedustega ning tehtud järeldusi mōnede aktiivsete võnkumiste iseloomu kohta. Polaarse lahusti môjju järgi $a$-klorofülli ja protoklorofülli spektrite struktuurile on leitud nende molekulide solvateerumismehhanismid erinevad olevat.

\section{k. AVARMAA, R. TAMKIVI, Sirje KIISLER, Vaimi NOMM \\ FINE-STRUCTURED VIBRONIC SPECTRA OF CHLOROPHYLL AND RELATED MOLECULES IN SOLID SOLUTIONS UNDER TUNABLE DYE LASER EXCITATION}

\begin{abstract}
Fluorescence and excitation spectra of frozen solutions of chlorophyll $a$, protochlorophyll, pheophytin $a$ and its $\mathrm{Zn}$-complex were measured at $4.2 \mathrm{~K}$, using tunable dye laser excitation. A number of vibrational frequencies of this group of related molecules was determined from the vibronic structure. The frequencies of different compounds were compared mutually, also with corresponding frequencies of phorphyrins, and conclusions about the character of some vibronically most active vibrations have been drawn. From the influence of the polar solvent on the structure of the spectra of chlorophyll $a$ and protochlorophyll, the solvation mechanisms for the two compounds were found to be different.
\end{abstract}

